\title{
BMJ Open Perfusion index in healthy newborns during critical congenital heart disease screening at 24 hours: retrospective observational study from the USA
}

\author{
Priya Jegatheesan, Matthew Nudelman, Keshav Goel, Dongli Song, \\ Balaji Govindaswami
}

To cite: Jegatheesan $P$, Nudelman M, Goel K, et al. Perfusion index in healthy newborns during critical congenital heart disease screening at 24 hours: retrospective observational study from the USA. BMJ Open 2017;7:e017580. doi:10.1136/ bmjopen-2017-017580

- Prepublication history for this paper is available online. To view these files please visit the journal online (http://dx.doi org/10.1136/bmjopen-2017017580).

Received 2 May 2017 Revised 28 August 2017 Accepted 29 August 2017
CrossMark

Department of Pediatrics, Newborn Medicine, Santa Clara Valley Health and Hospital System, San Jose, California, USA

Correspondence to Dr Priya Jegatheesan; Priya.Jegatheesan@hhs.sccgov. org

\section{ABSTRACT}

Objective To describe the distribution of perfusion index (PI) in asymptomatic newborns at 24 hours of life when screening for critical congenital heart disease (CCHD) using an automated data selection method.

Design This is a retrospective observational study. Setting Newborn nursery in a California public hospital with $\sim 3500$ deliveries annually.

Methods We developed an automated programme to select the PI values from CCHD screens. Included were term and late preterm infants who were screened for CCHD from November 2013 to January 2014 and from May 2015 to July 2015. PI measurements were downloaded every $2 \mathrm{~s}$ from the pulse oximeter and median $\mathrm{PI}$ were calculated for each oxygen saturation screen in our cohort.

Results We included data from 2768 oxygen saturation screens. Each screen had a median of 29 data points (IQR 17 to 49$)$. The median PI in our study cohort was $1.8(95 \%$ Cl 1.8 to 1.9) with IQR 1.2 to 2.7. The median preductal PI was significantly higher than the median postductal ( 1.9 vs $1.8, p=0.03$ ) although this difference may not be clinically significant.

Conclusion Using an automated data selection method, the median PI in asymptomatic newborns at 24 hours of life is 1.8 with a narrow IQR of 1.2 to 2.7. This automated data selection method may improve accuracy and precision compared with manual data collection method. Further studies are needed to establish external validity of this automated data selection method and its clinical application for CCHD screening.

\section{INTRODUCTION}

Critical congenital heart disease (CCHD) refers to a group of heart defects that require surgery or catheter-based intervention within the first year of life. Universal oxygen saturation screening of all newborns at 24 hours of life with pulse oximetry has been recommended and is now standard of care in many centres to improve recognition of CCHD in asymptomatic newborns. ${ }^{1-6}$ Despite this current screening, we are still unable to detect all CCHD cases prior to newborn

\section{Strengths and limitations of this study}

- This study describes a novel automated data selection method to calculate the median perfusion index (PI) from data downloaded directly from pulse oximeter.

- The automated data selection method used in this study potentially minimises data collection errors and bias.

- This study describes the distribution of $\mathrm{PI}$ in asymptomatic newborns at 24 hours during critical congenital heart disease screen.

- In this study, the PI data obtained from pulse oximeter were deidentified and therefore not directly linked to the clinical outcome of the infants.

- This was a single-centre study from a public hospital that serves a primarily Hispanic population.

hospital discharge, especially in lesions with left ventricular outflow obstruction. ${ }^{4-9}$

Perfusion index (PI) reflects the ratio of the pulsatile to non-pulsatile signal components of blood flow in the peripheral tissue. It is derived from the photoelectric plethysmographic signal of a pulse oximeter. PI thus non-invasively provides continuous real-time perfusion status of the selected monitoring site. ${ }^{10}{ }^{11}$ In recent years, PI has been suggested to have use as an adjunct to pulse oximetry screening to detect non-cyanotic CCHD cases. ${ }^{4}{ }^{12-14}$ Multiple studies have described PI values in the first few days of life in term and preterm infants. ${ }^{12}$ 15-19 A lower PI in neonates has been correlated with lower superior vena cava flow $^{20}$ and shown to be a predictor of illness severity and subclinical chorioamnionitis. ${ }^{21} 22$

Despite PI being a simple, non-invasive objective measurement with potential clinical applications, it has been underused. This may in part be due to the inherent variability in PI that is seen during a brief monitoring period. ${ }^{19}$ Values with variability that need to 
be manually collected are difficult to standardise. Using automated data selection methods may assist with standardised data collection. The objective of our study is to describe the distribution of PI in asymptomatic newborns at 24 hours of life when they undergo CCHD screening using pulse oximetry with an automated data selection method.

\section{METHODS}

\section{Design}

This is a retrospective observational study.

\section{Subjects and setting}

Data were collected directly from pulse oximeters used for CCHD screen in asymptomatic term and late preterm infants in the newborn nursery at a public hospital with $\sim 3500$ deliveries annually. Data collection was Institutional Review Board approved.

\section{CCHD screen}

All infants in the newborn nursery underwent CCHD screen at 24 hours of age using two motion-tolerant pulse oximeter monitors ( $\operatorname{Rad} 7$, Masimo Corporation, Irvine, California, USA) with an $8 \mathrm{~s}$ averaging time to measure both preductal and postductal oxygen saturation $\left(\mathrm{SpO}_{2}\right)$ simultaneously.

Reusable probes with disposable wraps were placed on the right palm or wrist for preductal and on either foot for postductal saturation. Nursing staff continued the pulse oximeter monitoring until they obtained stable tracings of the $\mathrm{SpO}_{2}$ and heart rate (HR) for the CCHD screen. While the $\mathrm{SpO}_{2}$ values were recorded by clinical nurses manually when both preductal and postductal pulse oximetry showed stable waveforms and the HRs were correlating, the machine recorded the values continuously. The screen was repeated if either the preductal and postductal saturations was $90 \%$ to $94 \%$, or there was a $>3 \%$ difference between the two values; $\mathrm{SpO}_{2}<90 \%$ was considered a failed screen and required immediate Neonatal Intensive Care Unit (NICU) evaluation. Median age when CCHD screen occurred in the nursery was 26.1 hours of life (IQR 24.8-28.3 hours). These pulse oximeters were used for CCHD screening and for repeat measurements done based on the screening protocol or nurse's clinical judgement in newborn nursery.

\section{Data collection}

Convenience sample was used based on the amount of data stored in the pulse oximeters. The values of PI measurements, $\mathrm{SpO}_{2}, \mathrm{HR}$, signal quality indicators, date and time of the screen were recorded every $2 \mathrm{~s}$. Deidentified data were downloaded using Trendcom software (Masimo Corporation, Irvine, California, USA). The screening data from the two pulse oximeters with identical time periods were used for analysis. Since the duration of recording for each screen varies, the total number of preductal and postductal screens recorded in the same time period were slightly different. The first screening dataset had recordings from November 2013 to January 2014 including both preductal and postductal data because the pulse oximeters were not selectively labelled as preductal or postductal. Pulse oximeters were subsequently labelled for the second dataset. The second dataset had non-paired preductal and postductal data recordings from May 2015 to July 2015.

\section{Data selection}

As a pilot study, we manually selected a subset of the PI data from the first dataset (222 screens; 7426 data values). For each individual screening data, the values from the point at which the $\mathrm{HR}$ and $\mathrm{SpO}_{2}$ had stabilised were included. Values with 'low $\mathrm{SpO}_{2}$ signal quality' (as preprogrammed in the pulse oximeter by the manufacturer) were eliminated. Subsequently, we developed an automated Excel program for data selection. The Excel program eliminated data rows without any numerical values recorded and then assigned unique dataset identifications based on the time intervals separating individual screens. Data points with a $\mathrm{HR}$ or a $\mathrm{SpO}_{2}$ equal to zero were excluded. Data points with low $\mathrm{SpO}_{2}$ signal quality were eliminated (figure 1). The Excel program was validated in the pilot study subset and then used to select the data points in the complete dataset for this study. ${ }^{67}$

\section{Data analysis}

Median PI was calculated for each individual screening data. The percentile distributions of the median PI values and their 95\% CIs were calculated for the unlabelled first study dataset and for the preductal and postductal PIs values in the second dataset separately. The median preductal and postductal PI in the second dataset were compared using the Mann-Whitney-Wilcoxon rank sum test. Statistical data analysis software STATA V.14.2 (StataCorp LP, College Station, Texas USA) was used for analysis.

\section{RESULTS}

The study dataset included data from 2768 screens. The median recording time per individual screen was $72 \mathrm{~s}$ (IQR 42-126s). The data selection process is outlined in (figure 1). Based on the automated program, 3.4\% of data points were eliminated for HR of zero, $0.3 \%$ for $\mathrm{SpO}_{2}$ of zero and $17.8 \%$ for low-signal quality. Of the 2768 screening data, only three screens $(0.1 \%)$ were eliminated based on the automated data selection process. Each screen had a median of 29 data points (IQR 17 to 49) after the data selection process. The median PI value in our whole study cohort was 1.8 (95\% CI 1.8 to 1.9$)$ with IQR 1.2 to 2.7 .

The percentile distribution of the median PIs in the first and second dataset are shown in (table 1). The distribution of the median PI for the unlabelled first dataset is shown in (figure 2). The second study dataset included 1352 screens, of which 682 and 670 were preductal and 


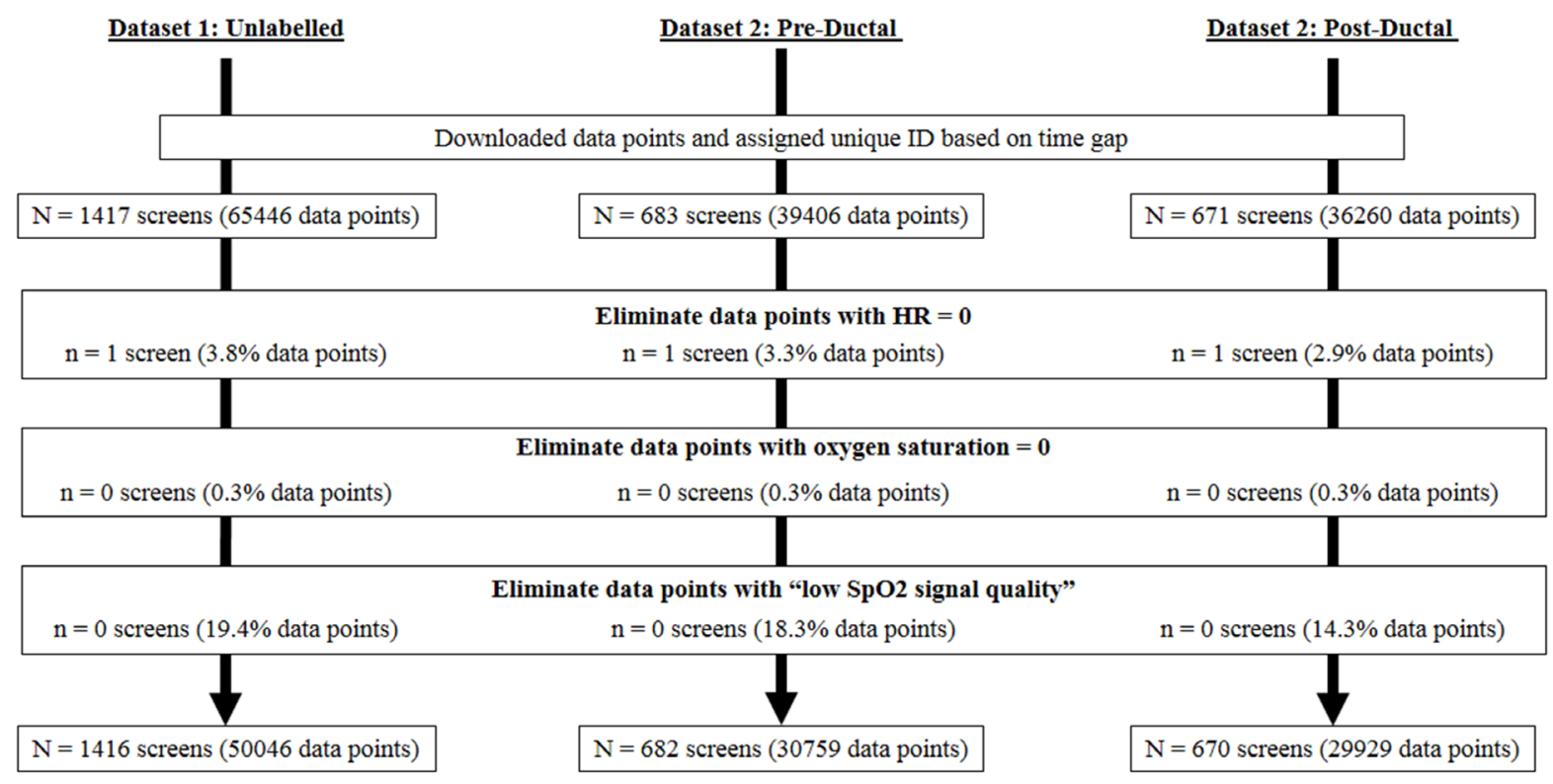

Figure 1 Data collection and automated data selection method.

postductal measurements, respectively. The median preductal PI was significantly higher than the median postductal (1.9 vs $1.8, \mathrm{p}=0.03$ ) although this difference may not be clinically significant. The distribution of the preductal and postductal median PIs are shown in (figure 3).

\section{DISCUSSION}

Prior to identifying cut-off values for PI to improve the detection of lesions with left ventricular outflow tract obstruction during CCHD screening, it is necessary to describe the normal PI distribution using a reliable automated method in asymptomatic infants at the time of screening. Although multiple studies have described PI in preterm and term newborns with various newborn conditions, ${ }^{7}{ }^{12}$ 14-24 few have described the PI distribution in asymptomatic term and late preterm infants, at 24 hours of life when CCHD screening using pulse oximetry is performed. Granelli et al described the distribution of preductal and postductal PI in 10000 normal healthy newborns prior to discharge, with a time of measurement ranging from 1 to 120 hours. ${ }^{12}$ Despite the difference in the age of measurement, the PI distribution in our cohort measured at 24 hours, median PI of 1.8 and IQR 1.2 to 2.7 , is almost identical to the values reported by Granelli et al, median PI of 1.7 and IQR 1.18 to 2.5. These two studies show that PI distribution is relatively consistent and stable in asymptomatic newborns during the CCHD screening time period in the first few days of life.

Table 1 Distribution of median PI

\begin{tabular}{lccc}
\hline & Unlabelled PI (n=1416) & Preductal PI (n=682) & Postductal PI (n=670) \\
\hline 1st \%ile & Median $(95 \% \mathrm{Cl})$ & Median $(95 \% \mathrm{Cl})$ & $\mathrm{Median} \mathrm{(95 \%} \mathrm{Cl)}$ \\
\hline 5th \%ile & $0.50(0.40$ to 0.60$)$ & $0.50(0.37$ to 0.54$)$ & $0.40(0.40$ to 0.50$)$ \\
\hline 25th \%ile & $0.70(0.65$ to 0.70$)$ & $0.70(0.70$ to 0.80$)$ & $1.60(0.60$ to 0.70$)$ \\
\hline 50th \%ile & $1.20(1.20$ to 1.30$)$ & $1.30(1.20$ to 1.30$)$ & $1.80(1.70$ to 1.90$)$ \\
75th \%ile & $1.80(1.80$ to 1.90$)$ & $1.90(1.80$ to 2.00$)$ & $2.60(2.50$ to 2.80$)$ \\
95th \%ile & $2.80(2.65$ to 2.91$)$ & $2.70(2.55$ to 2.90$)$ & $4.67(4.20$ to 5.33$)$ \\
99th \%ile & $5.90(5.46$ to 6.93$)$ & $5.67(4.80$ to 7.15$)$ & $8.16(6.25$ to 14.24$)$ \\
\hline
\end{tabular}

$\mathrm{PI}$, perfusion index. 

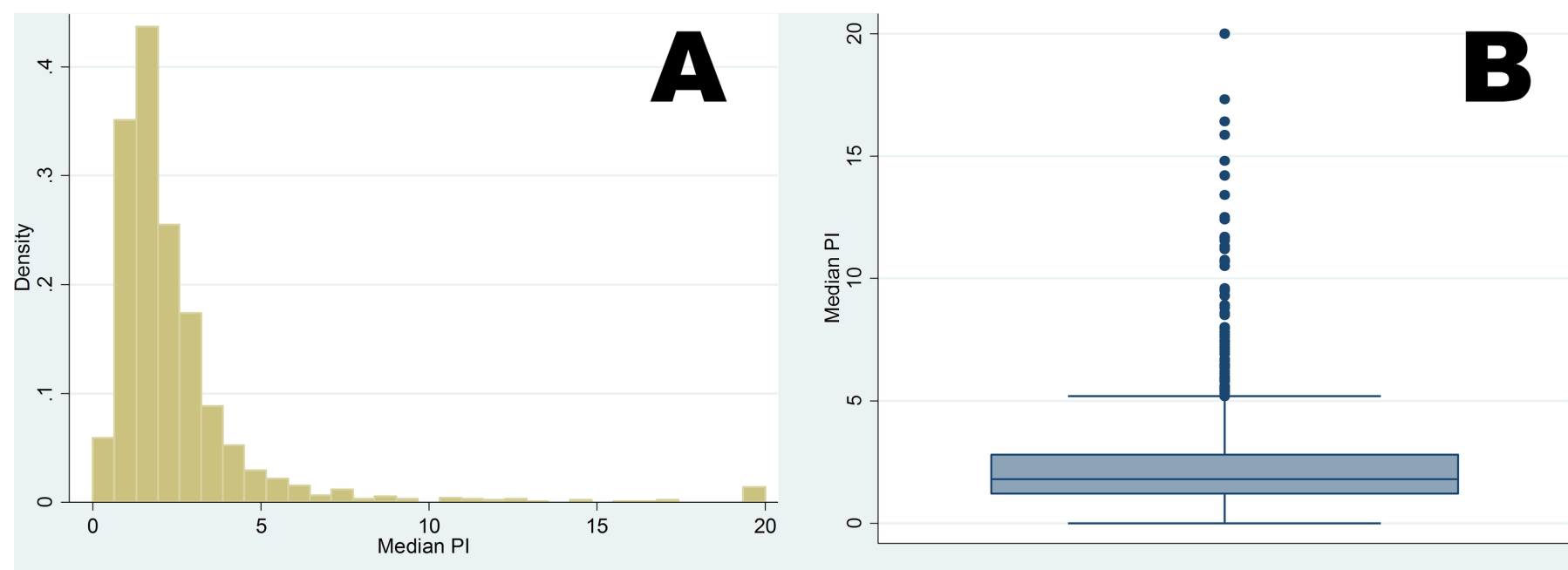

Figure 2 (A) Histogram of the median perfusion index. (B) Distribution of median perfusion index of the unlabeled dataset. The boxes represent the IQR from 25th-75th percentile (IQR). The marker within the box is the median and the 'whiskers' reach 1.5 times IQR.

A PI value of $<0.44$ has been shown to be indicative of low superior vena cava flow of $<40 \mathrm{~mL} / \mathrm{kg} / \mathrm{min}$ in very low birthweight infants. ${ }^{20}$ In a case-control study, Granelli et al suggested using a PI value of 0.7 as an adjunct to oxygen saturation screening to evaluate whether a newborn has left heart obstructive lesions. ${ }^{12}$ In our study, the first percentile PI value was 0.5 (95\% CI 0.40 to 0.60 ) and the fifth percentile PI value was 0.7 (95\% CI 0.65 to $0.70)$. A low PI value of $<0.5$ or $<0.7$ can be considered as a screening marker of hypoperfusion in the newborn, however, the sensitivity and specificity of such values to detect CCHD or other conditions is yet to be determined. A more recent study by Schena et al, in 42000 newborns, using a combined pulse oximeter and PI screen to detect CCHD, chose PI $<0.9$ as abnormal for their CCHD screen done between 48 and 72 hours of life. ${ }^{14}$ The one case of coarctation of aorta that was identified based on PI alone had a PI value of 0.3 . This study further showed that adding PI to pulse oximeter screens still missed two cases of left-sided obstructive CCHD. Hence, the clinical use of lower limit of PI in screening for CCHD in asymptomatic newborns needs further study.
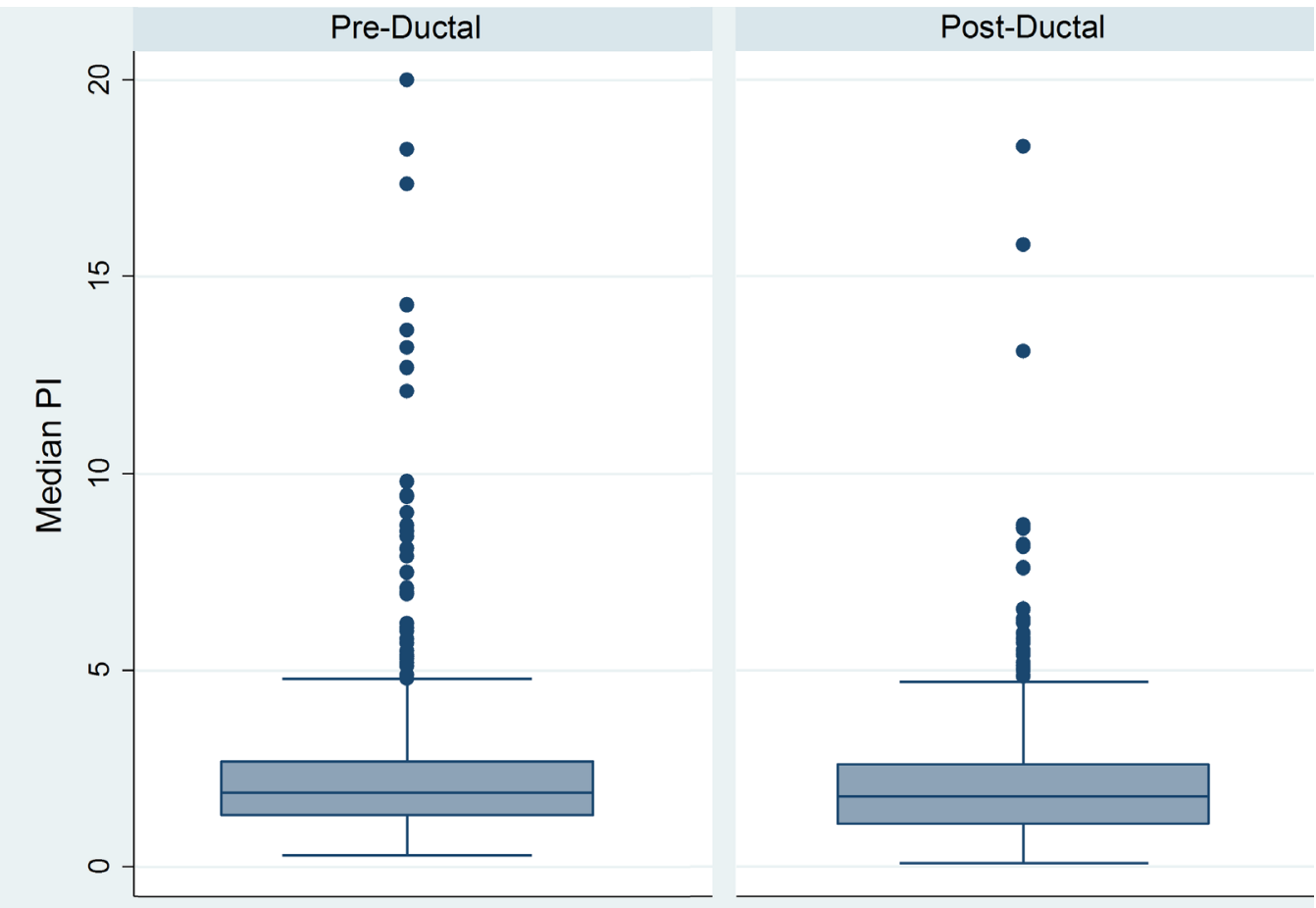

Figure 3 Distribution of preductal and postductal median perfusion index. The boxes represent the IQR from 25th-75th percentile. The marker within the box is the median and the 'whiskers' reach 1.5 times IQR. 
Our study demonstrates that at 24 hours postductal PI is lower than the preductal PI by 0.1 . Others have reported that postductal PI is higher than preductal PI. ${ }^{12}$ Granelli et al showed that the postductal PI is 0.02 higher than preductal PI. Such small differences in PI are not clinically significant in healthy newborns. However, this difference was much greater in Hakan's study of 196 healthy term infants that described median PI value and IQR for the paired preductal and postductal as $1.35(1.02-1.91)$ and $0.88(0.62-1.22)$ in the first day of life, and the difference was seen up to 3 days of life. ${ }^{18}$ The reason for the discrepancy in the preductal and postductal PI differences among these three studies is unclear. There are differences in methodology between our study and the other two studies. We screened newborns at 24 hours, Granelli et al screened between 1 and 120 hours and Hakan et al screened at 'first day' but the hour of life is unspecified. While our study compared the overall distribution of the preductal and postductal PI, the other two studies used paired preductal and postductal data samples. We should consider collecting and evaluating the preductal and postductal PIs in a larger patient population to further improve CCHD screening methodology.

It is common to have PI values fluctuate as they reflect a brief momentary measurement of one's peripheral perfusion at the site of the pulse oximeter. Peripheral perfusion is dynamically affected by a variety of factors. For this reason, a single transient measurement has limited value because it may not accurately reflect one's true perfusion status. Instead, the median PI calculated from monitoring PI over a period of time after the pulse oximeter tracing stabilises may provide a more accurate depiction of true perfusion status. ${ }^{13}$ Our study recorded PI for a median of $72 \mathrm{~s}$. As an additional step to ensure measurement accuracy, we used an automated (Excel-based) data selection process to capture only artefact-free PI values with correspondingly stable $\mathrm{SpO}_{2}$ and HR. Even though almost 20\% (3\%-4\% due to zero HR/saturation; $18 \%$ due to low signal quality) of the data points were eliminated by this method, we only lost $0.1 \%$ of the individual screening data. Other studies have used a similar methodology of selecting the median PI of artefact-free readings instead of using a single PI value. ${ }^{1518} 19$ Using electronic data output and data selection methods to standardise practice and eliminate human transcription error is essential in evaluating the efficacy of different PI values and their clinical significance. The data selection methods used in this and other studies will help to develop algorithms for automated PI data selection and output for clinical use.

A limitation of our study is that it is a retrospective single-centre study and the data were deidentified. We do not have paired samples to evaluate the individual preductal and postductal differences. We are unable to link PI data directly to clinical outcome of these infants. However, we have a prospectively maintained NICU database which allowed us to confirm that during the study period, there were no CCHD cases or early onset bacterial sepsis or pneumonia identified in the newborn nursery prior to discharge. The majority of infants born in our hospital continue to obtain care within our hospital system. Our hospital database provided sufficient follow-up information to determine whether any infant discharged during the study period was later diagnosed with CCHD. An exception would be if an infant was later diagnosed with CCHD at a different hospital.

The generalisability of our study is limited to the availability of the software to download data from the pulse oximeters. Once the data are downloaded, our automated data selection process can be reproduced easily. It is now standard of care to screen most newborns in the USA at 24 hours of life for CCHD. Centres using pulse oximeters that have numerical PI capability should be able to replicate our results. Generalisability for clinical use requires development of an automated algorithm in the pulse oximeters to provide the median PI value as output.

\section{CONCLUSION}

The median PI calculated using an automated data selection method in asymptomatic newborns at 24 hours of life is 1.8 , with a narrow IQR of 1.2 to 2.7. This distribution of PI is consistent with the previously published Swedish nomogram (median 1.7 and IQR 1.2, 2.5) described at 1-120 hours of life. This suggests that the PI values in asymptomatic newborns have a narrow range of distribution during the time of CCHD screening. Further studies are needed to establish external validity of this automated data selection method and its clinical application for CCHD screening.

Acknowledgements We acknowledge patients and their families. We also thank the postpartum unit nurses and hospital staff who played a major role in this study.

Contributors PJ, MN, DS and BG conceptualised and designed the study, critically reviewed and revised the manuscript. KG performed the pilot study with validation of the data selection process and assisted with drafting the initial abstract. All authors approved the final manuscript as submitted and agree to be accountable for all aspects of the work.

Funding The authors received the data download software from the company for the purpose of research.

Disclaimer There are no prior publications or submissions with any overlapping information, including studies and patients. This manuscript has not been and will not be submitted to any other journal while it is under consideration by BMJ Paediatrics. Authors have indicated they have no financial relationships relevant to this article to disclose. Each author listed on the manuscript has seen and approved the submission of this manuscript version and takes full responsibility for the manuscript.

Competing interests None declared.

Ethics approval Santa Clara Valley Health and Hospital System, Institutional Review Board.

Provenance and peer review Not commissioned; externally peer reviewed.

Data sharing statement The dataset is available from the Dryad repository, DOl: $10.5061 /$ dryad.bs935 Also is available for review by contacting the author at priya. jegatheesan@hhs.sccgov.org.

Open Access This is an Open Access article distributed in accordance with the Creative Commons Attribution Non Commercial (CC BY-NC 4.0) license, which permits others to distribute, remix, adapt, build upon this work non-commercially, 
and license their derivative works on different terms, provided the original work is properly cited and the use is non-commercial. See: http://creativecommons.org/ licenses/by-nc/4.0/

(c) Article author(s) (or their employer(s) unless otherwise stated in the text of the article) 2017. All rights reserved. No commercial use is permitted unless otherwise expressly granted.

\section{REFERENCES}

1. Mahle WT, Newburger JW, Matherne GP, et al. Role of pulse oximetry in examining newborns for congenital heart disease: a scientific statement from the AHA and AAP. Pediatrics 2009;124:823-36.

2. Hoffman Jl. It is time for routine neonatal screening by pulse oximetry. Neonatology 2011;99:1-9.

3. Kemper AR, Mahle WT, Martin GR, et al. Strategies for implementing screening for critical congenital heart disease. Pediatrics 2011;128:e1 259-e1267.

4. Ewer AK, Furmston AT, Middleton LJ, et al. Pulse oximetry as a screening test for congenital heart defects in newborn infants: a test accuracy study with evaluation of acceptability and costeffectiveness. Health Technol Assess 2012;16:1-184.

5. Mahle WT, Martin GR, Beekman RH, et al. Endorsement of health and human services recommendation for pulse oximetry screening for critical congenital heart disease. Pediatrics 2012;129:190-2.

6. Thangaratinam S, Brown K, Zamora J, et al. Pulse oximetry screening for critical congenital heart defects in asymptomatic newborn babies: a systematic review and meta-analysis. Lancet 2012;379:2459-64.

7. de-Wahl Granelli A, Wennergren M, Sandberg K, et al. Impact of pulse oximetry screening on the detection of duct dependent congenital heart disease: a Swedish prospective screening study in 39,821 newborns. BMJ 2009;338:a3037.

8. Govindaswami B, Jegatheesan P, Song D. Oxygen Saturation Screening for Critical Congenital Heart Disease. Neoreviews 2012;13:e724-e731.

9. Ailes EC, Gilboa SM, Honein MA, et al. Estimated number of infants detected and missed by critical congenital heart defect screening. Pediatrics 2015;135:1000-8.
10. Lima AP, Beelen P, Bakker J. Use of a peripheral perfusion index derived from the pulse oximetry signal as a noninvasive indicator of perfusion. Crit Care Med 2002;30:1210-3.

11. Lima A, Bakker J. Noninvasive monitoring of peripheral perfusion. Intensive Care Med 2005;31:1316-26.

12. Granelli A, Ostman-Smith I. Noninvasive peripheral perfusion index as a possible tool for screening for critical left heart obstruction. Acta Paediatr 2007;96:1455-9.

13. Piasek CZ, Van Bel F, Sola A. Perfusion index in newborn infants: a noninvasive tool for neonatal monitoring. Acta Paediatr 2014;103:468-73.

14. Schena F, Picciolli I, Agosti M, et al. Perfusion index and pulse oximetry screening for congenital heart defects. J Pediatr 2017;183:74-9.

15. Cresi F, Pelle $\mathrm{E}$, Calabrese $\mathrm{R}$, et al. Perfusion index variations in clinically and hemodynamically stable preterm newborns in the first week of life. Ital J Pediatr 2010;36:6.

16. Vidal $\mathrm{M}$, Ferragu $\mathrm{F}$, Durand $\mathrm{S}$, et al. Perfusion index and its dynamic changes in preterm neonates with patent ductus arteriosus. Acta Paediatr 2013;102:373-8.

17. Kinoshita M, Hawkes CP, Ryan CA, et al. Perfusion index in the very preterm infant. Acta Paediatr 2013;102:e398-e401.

18. Hakan N, Dilli D, Zenciroglu A, et al. Reference values of perfusion indices in hemodynamically stable newborns during the early neonatal period. Eur J Pediatr 2014;173:597-602.

19. Kroese JK, van Vonderen JJ, Narayen IC, et al. The perfusion index of healthy term infants during transition at birth. Eur $J$ Pediatr 2016;175:475-9.

20. Takahashi S, Kakiuchi S, Nanba Y, et al. The perfusion index derived from a pulse oximeter for predicting low superior vena cava flow in very low birth weight infants. J Perinatol 2010;30:265-9.

21. De Felice $C$, Latini $G$, Vacca P, et al. The pulse oximeter perfusion index as a predictor for high illness severity in neonates. Eur $J$ Pediatr 2002;161:561-2.

22. De Felice C, Del Vecchio A, Criscuolo M, et al. Early postnatal changes in the perfusion index in term newborns with subclinical chorioamnionitis. Arch Dis Child Fetal Neonatal Ed 2005;90:F411-F414.

23. Hawkes GA, O'Toole JM, Kenosi M, et al. Perfusion index in the preterm infant immediately after birth. Early Hum Dev 2015;91:463-5.

24. De Felice C, Goldstein MR, Parrini S, et al. Early dynamic changes in pulse oximetry signals in preterm newborns with histologic chorioamnionitis. Pediatr Crit Care Med 2006;7:138-42. 